\title{
Reflets
}

Revue ontaroise d'intervention sociale et communautaire

\section{Parole aux jeunes : ils nous disent ce qu'il faut pour assurer le succès du placement résidentiel}

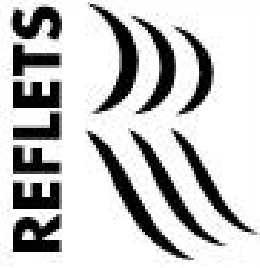

\section{Beverly Ann Byrne et Raymond Lemay}

Volume 11, numéro 1, 2005

Exclusion sociale

URI : https://id.erudit.org/iderudit/013066ar

DOI : https://doi.org/10.7202/013066ar

Aller au sommaire du numéro

Éditeur(s)

Reflets : Revue ontaroise d'intervention sociale et communautaire

ISSN

1203-4576 (imprimé)

1712-8498 (numérique)

Découvrir la revue

Citer cet article

Byrne, B. A. \& Lemay, R. (2005). Parole aux jeunes : ils nous disent ce qu'il faut pour assurer le succès du placement résidentiel. Reflets, 11(1), 196-213.

https://doi.org/10.7202/013066ar

Tous droits réservés (C) Reflets : Revue ontaroise d'intervention sociale et communautaire, 2005
Ce document est protégé par la loi sur le droit d'auteur. L'utilisation des services d'Érudit (y compris la reproduction) est assujettie à sa politique d'utilisation que vous pouvez consulter en ligne.

https://apropos.erudit.org/fr/usagers/politique-dutilisation/ 


\section{Parole aux jeunes : ils nous disent ce qu'il faut pour assurer le succès du placement résidentiel}

Beverly Ann Byrne et Raymond Lemay

Services aux enfants et adultes de Prescott-Russell

La maltraitance des enfants est très répandue; en Ontario, en 1993, il y a eu approximativement 12600 enfants victimes de maltraitance (Trocmé, McPhee et Kwan Tam 1995 : 569). Les conséquences de ce mauvais traitement sont dévastatrices pour l'enfant de même que pour l'adolescent (Hanigan 1990; NRC 1993; Starr 1988) et peuvent entraîner une séquence d'événements négatifs qui s'étendra jusque dans la vie adulte (Seligman 1993). Par sa Loi sur les services à l'enfance et à la famille, le gouvernement ontarien s'engage à protéger les enfants et à assurer leur bienêtre (Ontario 1985:8). Lorsque les services directs à une famille biologique ne suffisent plus pour assurer la protection des enfants, une société de l'aide à l'enfance (SAE) peut mener une intervention de dernier recours, c'est-à-dire le placement temporaire dans un foyer substitut.

En 2003, environ 17000 enfants ontariens étaient placés sous les soins des services de bien-être à l'enfance. 53,4\% des enfants pris en charge par la SAE étaient âgés de 13 à 21 ans (Canada 1994 : 102). Selon la législation ontarienne, l'objectif du placement vise la croissance et le développement de l'enfant dans un environnement stable qui devra répondre aux besoins particuliers de l'enfant jusqu'à ce qu'une solution permanente soit trouvée (Ontario 1985: 9 standards). 
"L'opinion des jeunes visés offrirait une perspective intéressante sur les difficultés vécues par des jeunes placés à long terme, mais surtout sur les éléments qui favorisent leur bien-être. »
Malheureusement, les résultats des jeunes issus de ce système résidentiel sont souvent négatifs, entre autres sur le plan scolaire et sur ceux de l'emploi et de l'indépendance financière (Broad 1999; Cook 1994). Ainsi, malgré les intentions des organismes de protection de l'enfance de veiller au mieux-être des enfants et adolescents, de nombreuses difficultés empêchent de rencontrer les objectifs de la loi (Baker 1995 : 245; Mckenzie 1994 :2).

La présente étude ${ }^{1}$ propose d'explorer l'expérience du placement selon la perspective des adolescents. Très peu de recherches ont étudié le placement selon le point de vue des jeunes qui vivent à long terme en foyer d'accueil. Pourtant, la perspective et la satisfaction des usagers ${ }^{2}$ influenceront la réussite des interventions (Bush et al. 1977; Chubb et Fertman 1992; Korbin 1977; Shulman 1993). L'opinion des jeunes visés offrirait une perspective intéressante sur les difficultés vécues par des jeunes placés à long terme, mais surtout sur les éléments qui favorisent leur bien-être. Dans leur annotation de la recherche sur la relation d'aide, Miller, Duncan et Hubble (1999) ont trouvé que la perception des clients ne correspond pas toujours à celle des intervenants qui travaillent avec eux. De plus, ces mêmes auteurs, de même que Bachelor et Horvath (1999) suggèrent que c'est la perception des clients qui prédit le mieux le succès (ou l'échec) éventuel de l'intervention. Ainsi, l'expertise de ces jeunes vient compléter l'expertise professionnelle (Le Bossé 1996 : 132).

Cette recherche vise donc à cerner les éléments qui, selon les jeunes placés en foyer d'accueil, favorisent leur bien-être. Leurs témoignages suggèrent quelques pistes d'intervention permettant à la SAE d'améliorer son intervention et ainsi favoriser le bienêtre de ces jeunes.

\section{Méthodologie}

Les participants à cette étude ont été recrutés parmi des jeunes sous la responsabilité d'une SAE de l'Est ontarien. L'échantillon a été constitué en fonction des critères suivants : jeunes francophones 
âgés de 14 à 21 ans, placés en famille d'accueil ou en foyer de groupe pour des motifs de maltraitance, depuis au moins un an et demi avec cette SAE.

Trente-sept jeunes placés à long terme en famille d'accueil ou en foyer de groupe ont été contactés. Un total de 11 jeunes dont 8 filles et 3 garçons ont accepté de participer à cette étude. Il s'agit d'un échantillon volontaire.

La collecte des données s'est effectuée au moyen de groupes d'entretien. Chaque participant a assisté à une seule rencontre de groupe d'une durée approximative de deux heures. Chaque groupe était composé de deux à cinq jeunes. Pendant les rencontres de groupe, les participants se sont prononcés sur divers sujets en rapport avec leur expérience en tant que jeune placé à long terme en foyer d'accueil. L'analyse des données en est une de contenu thématique et le guide d'entrevue représente une partie de la grille d'analyse (Mayer et Ouellet 1991 : 82; Patton 1990 :376).

\section{Constats préliminaires}

"L'élément le plus notable lors de l'analyse des entretiens de groupe a été l'importance accordée par les jeunes aux intervenants impliqués dans leur vie : les travailleurs sociaux, les parents d'accueil, le personnel des foyers de groupe et les enseignants. "
L'élément le plus notable lors de l'analyse des entretiens de groupe a été l'importance accordée par les jeunes aux intervenants impliqués dans leur vie: les travailleurs sociaux, les parents d'accueil, le personnel des foyers de groupe et les enseignants. Les jeunes semblent avoir davantage de choses à dire sur les caractéristiques, les attitudes et les interventions des personnes qui les entourent que sur les structures encadrant ces personnes, ce qui confirme les propos de Hubble, Duncan et Miller (1999) selon lesquels les sciences de l'homme reconnaissent toutes qu'un instrument très important de l'intervention est la personne même de l'intervenant.

Selon notre analyse des propos des jeunes répondants, les principaux éléments identifiés comme étant favorables au bienêtre de ces jeunes sont: les caractéristiques individuelles des personnes impliquées dans leur vie, la stabilité, la préparation au 
placement et au déplacement et l'implication des jeunes dans la prise de décisions.

Un élément a été perçu par les participantes et les participants comme étant négatif pour leur bien-être :l'étiquette " enfant placé ». Cet élément est inclus dans cette analyse, car il a été énoncé à plusieurs reprises. Chaque énoncé sera suivi d'extraits des entretiens de groupes considérés comme un corpus global.

En somme, donnons la parole aux jeunes. Leurs propos nous permettront de bonifier nos interventions auprès d'eux.

\section{Résultats et discussion}

\section{Les caractéristiques personnelles}

Malgré les rôles différents assumés par les intervenants, les parents d'accueil et le personnel des foyers de groupe, les jeunes semblent rechercher les mêmes attributs chez chacun. Parmi ceux perçus comme étant favorables à leur bien-être, l'on retrouve l'écoute, l'aide concrète, un comportement énergique et une attitude positive à leur égard. Selon les jeunes rencontrés, ces caractéristiques sont porteuses d'impact et de succès.

\section{L'écoute}

Elle est une habileté importante en service social (Duncan \& Miller 2000). Les jeunes en notent l'importance même dans un contexte non thérapeutique. En vieillissant, ils cherchent de plus en plus les occasions de développer leurs capacités de penser, de discuter et de prendre des décisions. Ils doivent se sentir écoutés.

Ainsi les jeunes nous disent :

"Il [le travailleur social] écoute tes opinions. 》 — "Ils [les parents d'accueil] sont là pour m'écouter. »— «Je peux leur [les enseignants] parler de n'importe quoi.» 
"Les témoignages des jeunes indiquent que l'écoute renvoie le message que ce qu'ils disent est important, et qu'ils sont eux-mêmes importants. »
$\mathrm{Au}$ contraire, un manque d'écoute frustre ces jeunes qui veulent tant s'exprimer et se faire entendre :

$$
\begin{aligned}
& \text { "Elle parle comme si je n'étais pas là. »— " Quand } \\
& \text { je fais un point, elle me coupe et me dit j'ai tort. " }
\end{aligned}
$$

Les témoignages des jeunes indiquent que l'écoute renvoie le message que ce qu'ils disent est important, et qu'ils sont euxmêmes importants.

\section{La sollicitude}

Selon ces jeunes, la perception d'une sollicitude sincère est un élément qui influence de façon positive la relation d'aide. Les jeunes ont besoin de saisir que les personnes impliquées dans leur vie sont présentes, qu'elles se préoccupent réellement d'eux. Toutefois, il importe de retenir que c'est la perception qu'a le jeune du niveau de préoccupation qui va influencer son bien-être (Shulman 1993 :96).Ainsi, il faut tenir compte de ce que les jeunes considèrent comme des indicateurs de sollicitude. Selon l'analyse des données, la sollicitude sincère des intervenants se caractérise par les gestes qui dépassent les tâches habituelles requises par leur emploi. Le dépassement des tâches exigées a été mis en valeur dans une étude précédente (Magura 1982).

"She cares. Elle n'est pas là juste parce qu'elle se fait payer." — "Ils travaillent pour moi et les autres jeunes au lieu de travailler pour eux-mêmes ou pour leurs boss." - "Qui fait plus que ce que ses tâches demandent. » - "Il est venu me voir sur son temps personnel. Ça m'a mis joyeuse. Ça m'a dit qu'il est vraiment là."

\section{L'attitude positive}

L'attitude positive et le niveau d'énergie de l'intervenant peuvent affecter l'humeur du jeune. L'attitude positive peut vraisemblablement correspondre à la volumineuse littérature sur les attentes positives qui jouent un rôle de premier plan dans 
l'intervention (Snyder, Michael \& Cheavens (1999), et même figurer comme facteur qui déterminera le succès de l'intervention (Kirsch 1999).

"Quelqu'un de super énergique. » — "Il joke et il rit.» — "Ils sont jeunes, donc ils savent ce qui se passe. »

Les participants nous indiquent que le contraire est aussi vrai : si une personne est continuellement négative, les émotions ressenties par les jeunes seront plus négatives. Une des adolescentes nous exprime que même les petits messages négatifs s'accumulent et ont un effet négatif sur son bien-être.

"Elle n'a rien de bon à dire. [...] Elle chiale. Ça fait que je pars le matin de chez moi puis je reviens le soir me recoucher. Je ne suis jamais là. » — "Quand les parents sont négatifs [...], tous les messages négatifs vont derrière la tête et it catches up with you. Tu commences à te poser des questions sur la vie, sur n'importe quoi et t'as toujours un côté noir. Ce n'est pas bon ça. »

\section{L'aide concrète}

Les jeunes ont souligné l'aide concrète comme étant un appui à leur bien-être. Ils doivent percevoir l'utilité de l'aide prodiguée dans leur quotidien. Ainsi, les conseils, les recommandations et les gestes qu'ils reçoivent en guise de soutien à leur propre prise en charge sont tous des exemples d'aide concrète qu'ils jugent favorables à leur bien-être.

"Ils peuvent fournir des informations [psychologue, choix de carrière ou autre]. " - "Quelqu'un [personnel de foyer de groupe] qui offre de l'aide concrète : aide à faire mon plan, trouve du counselling et $d u$ soutien pour finir mes études. » — "Il m'a aidé en me donnant des conseils sur la gestion de mon temps. » 
Encore une fois, nous voyons comment la perception des jeunes va influencer les résultats de l'intervention (Korbin 1977; Bush et al. 1977).

\section{La disponibilité}

Elle a été mentionnée par les jeunes comme étant un élément qui influence leur bien-être. Ils en ont mentionné l'importance uniquement par rapport aux travailleurs sociaux. Les éléments qui indiquent aux jeunes la disponibilité sont, par exemple, le retour d'un appel de façon prompte ou simplement savoir que le travailleur sera là en cas de besoin.

"Il m'a dit que je pouvais l'appeler n'importe quand si j'avais des problèmes. Ça me rassure." — "Je peux la rejoindre si j'ai besoin.» — "Savoir que quelqu'un est là. [Ça l'affecte] ta sécurité; savoir que tu es sécure."

Le manque de disponibilité semble provoquer une certaine inquiétude chez les jeunes. En effet, la personne sur qui ils dépendent n'est pas toujours disponible pour les aider.

"[...] d'être là quand on en a de besoin parce que des fois, c'est vraiment important; puis ils ne sont pas là ou leur answering machine n'est pas à date. » — «Par exemple, si les parents à M. me jettent dehors, je vais appeler ma worker parce que c'est mon parent. Une semaine et demie [avant qu'elle me rappelle] où je vais rester pendant une semaine et demie? Qu'est-ce que je vais faire? C'est pour ta sécurité; savoir qu'il y a quelqu'un là. Savoir que tu es sécure. Tu as besoin de savoir ça. Tu as besoin de savoir que si tu les appelles, ils vont te rappeler."

Les participants transmettent clairement l'importance d'avoir accès à une personne disponible à répondre à leurs besoins. Les jeunes voient la disponibilité comme un élément de sécurité, particulièrement en situation d'urgence. Ils soulignent l'importance 
pour le travailleur représentant le parent corporatif (SAE) de se rendre disponible pour répondre à leurs besoins.

\section{Le manque de temps}

Les jeunes formulent des hypothèses pour expliquer les manquements individuels mentionnés précédemment tels que les manques d'écoute et de disponibilité chez les travailleurs sociaux. Ils saisissent que les travailleurs sociaux en protection de l'enfance n'ont tout simplement pas le temps de s'occuper d'eux. Ils ont une charge de travail très élevée et il est difficile de trouver le temps requis pour favoriser le bien-être du jeune.

"Ils prennent trop de temps pour remplir leurs papiers qu'ils n'ont pas le temps de faire rien d'autre." — "Elle m'appelle seulement lorsque je dois remplir des papiers. " — "Des fois, je trouve qu'ils vont trop vite et je n'ai pas le temps de répondre. Tu ne veux pas parler parce que si tu ne peux même pas transmettre tout ton point de vue...»

Ces témoignages viennent alimenter l'importance d'afficher une préoccupation sincère de la part des intervenants. Les jeunes veulent être considérés comme des personnes plutôt que comme les pendants de tâches bureaucratiques. Ils veulent être la priorité. Ces propos rappellent la raison d'être de l'organisme : « d'œuvrer dans l'intérêt des enfants et d'assurer leur protection et leur bienêtre " (Ontario $1985: 8$ ).

\section{La stabilité}

Un aspect important de l'intervention à la SAE est celui d'assurer la stabilité dans plusieurs aspects de la vie d'un enfant : environnement, relations familiales et sociales, milieu scolaire. (Berry et Pelton 1994 : 262; Ontario 1985 : 19). Cette stabilité des relations avec l'environnement est un ingrédient fondamental pour assurer le développement positif de l'enfant et de l'adolescent (Goldstein et al. 1978; Kufelt et al 1995). Les jeunes ont identifié deux types de stabilité : celle des travailleurs sociaux et celle du placement. 


\section{La stabilité des travailleurs sociaux}

La stabilité des relations avec les personnes significatives est importante dans la vie d'un enfant et d'un adolescent (Bourgeault et de la Harpe 1988; Goldstein et al.1978). Selon les jeunes, la stabilité des travailleurs est importante, car elle facilite l'établissement d'une bonne relation entre eux et le travailleur, ce qui facilite le travail qu'ils ont à accomplir ensemble (Magura 1982; Shulman 1993). Les propos suivants illustrent l'importance de la stabilité :

"Ils savent de quoi tu parles. "— « Elle sait comment je suis [...] Il $y$ a moins de confusion et de perte de temps. " — "Comme moi, ça fait trois ans que je suis avec le même travailleur. Je le connais bien et je sais comment il marche."

Les jeunes ont énuméré certains effets néfastes que provoquent, sur leur bien-être, les changements fréquents de travailleurs sociaux. Ils les résument ainsi :

"On doit toujours recommencer l'histoire et ça fait mal. » — "Comme quand tu la rencontres en premier, il faut que tu établisses une relation. Si tu changes tout le temps, il faut que tu recommences encore et encore, tu ne peux pas grandir."

\section{La stabilité du placement}

Bien que celle-ci n'a pas été mentionnée spécifiquement comme étant un élément favorable à leur bien-être, son contraire, l'instabilité des placements, a des effets néfastes, selon les jeunes. Voici ce qu'ils en disent :

"Tu ne peux pas t'attacher et t'as moins de sécurité... ça te mélange toute." - "Je trouve ça difficile de déménager... ne pas savoir ou tu t'en vas, qu'est-ce qui va se passer, c'est qui ça? Plein de questions." — "J'ai changé d'école tellement de fois. Les autres sont plus avancés que moi et c'est gênant." 
Les participants ont de plus mentionné que la familiarité des parents d'accueil, du personnel du foyer et de l'environnement était un élément propice à leur bien-être. Puisque la familiarité découle de la stabilité, il est possible d'en induire que, selon les jeunes, la stabilité favorise leur bien-être grâce à cette familiarité.

"Je connais l'environnement. » — «Ils connaissaient ma mère donc je peux leur parler de nos chicanes et ils comprennent. " - "Je connaissais des personnes où je déménageais. Ça l'a aidé."

Un adolescent nous fait part de son mécontentement par rapport au manque de familiarité :

"Je ne suis pas bien dans cette famille. Je viens de Vanier et j'habite maintenant à Orléans. Je ne connais personne."

Bref, la stabilité des travailleurs sociaux est importante pour les jeunes puisqu'elle permet la création d'un lien et une connaissance plus profonde l'un de l'autre; cela favorise la relation de confiance ainsi que la relation d'aide. Les parents naturels étant absents, la stabilité des travailleurs sociaux et la stabilité du placement sont d'autant plus importantes quand ces personnes deviennent celles qui assurent la sécurité affective.

\section{La préparation au placement}

Selon Steinhauer (1991), la préparation est un des facteurs qui influencent la réussite du placement et, par voie de conséquence, le bien-être des jeunes. La préparation est encore plus importante quand les jeunes vivent des déplacements multiples. Selon les jeunes, cette préparation est importante, car elle les sécurise.

"Mon t.s. m'a dit où c'était et il m'a préparé."

— "C'est rassurant de savoir ce qu'il va t'arriver dans la prochaine place."

D'un autre côté, le manque de préparation semble créer de l'anxiété face à l'inconnu. Les jeunes qui n'ont pas été bien 
préparés avant un placement transmettent de façon claire les effets néfastes que cela implique, comme l'insécurité et la peur.

"Je n'ai pas eu de préparation. Je pensais que je m'en allais en prison. Ça m'a fait peur. J'ai fait une grosse crise, car je me demandais où je m'en allais. » — " On ne m'a pas dit où je m'en allais. J'ai juste paqueté mes choses puis ils m'ont dit: tu t'en vas ailleurs! Je ne savais pas où je m'en allais, ni pourquoi."

La préparation, ou le manque de préparation, a des effets à deux niveaux. Premièrement, les jeunes nous disent que, lors d'un changement, la préparation peut augmenter ou minimiser les sentiments d'anxiété et de peur. Les données recueillies indiquent que les jeunes ont besoin du temps pour s'adapter au changement et qu'ils ont aussi besoin de comprendre les raisons du changement. Une étude effectuée par Johnson,Yoken etVoss (1995) confirme les mêmes informations : les jeunes désirent avoir plus d'information lors d'un placement ou d'un déplacement. Deuxièmement, les écrits consultés nous indiquent que la préparation a une incidence sur l'échec ou sur la réussite du placement (Steinhauer 1991). Ainsi, est-elle d'une importance cruciale dans la vie d'un enfant placé sous les soins d'un organisme de protection comme la SAE. L'implication du jeune dans les décisions prises avant le placement ou le déplacement fait partie de la préparation.

\section{L'implication des jeunes}

Un quatrième élément que les jeunes ont identifié comme étant favorable à leur bien-être est leur implication dans les décisions

"Les jeunes désirent être consultés et avoir un sentiment de contrôle sur leur situation. » et les démarches prises à leur égard. Les jeunes désirent être consultés et avoir un sentiment de contrôle sur leur situation. D'autres recherches soulignent également le désir exprimé par les jeunes et l'importance qu'ils accordent à être impliqués dans les décisions qui les concernent (Bush, Gordon et LeBailly 1977 : 496; Taber et Proch 1987). L'adolescence est une période pendant laquelle les capacités intellectuelles du jeune se développent pour rejoindre celles d'un adulte (Bélanger 1987). Ainsi, le jeune 
devient de plus en plus apte à se questionner et à faire des choix sur les différentes situations qui lui sont présentées (Bélanger 1987 : 109). L'implication du jeune signifie la possibilité pour lui de participer aux événements qui le touchent, d'émettre ses idées et ses opinions ainsi que de jouir du privilège de certains choix. De plus, c'est un élément important pour le développement de l'autonomie (Bélanger 1981 : 20).

"Il a vraiment tenu compte de mon opinion sur la famille d'accueil. Ça m'a fait du bien." - «On devrait avoir le choix d'où on va être placé. " — "J'ai toujours été en contrôle des décisions qui se faisaient au sujet de mes placements. " — "C'est rassurant de savoir que t'as le choix. [Si t'as le choix entre deux ou trois placements]."

Les jeunes expriment un sentiment d'impuissance lorsqu'ils n'ont pas le choix ou la liberté d'opinion sur leur propre situation.

"Vous aimez ça qu'on vous demande votre opinion?"

— "Se sentir égale à la personne qui travaille avec toi sinon ça ne marche pas, car il y a une personne qui overpowers et l'autre est prise."

Les jeunes placés en foyer d'accueil, plus particulièrement ceux qui ont vécu plusieurs changements de foyers, se sentent parfois impuissants devant des situations qui les affectent. Donc, les impliquer dans le processus vient compenser; c'est un élément important pour les jeunes non seulement sur ce qui entoure le placement, mais aussi sur différents secteurs de leur vie : l'école, les loisirs, les relations avec les adultes. Le nombre restreint de foyers d'accueil fait en sorte qu'il est rarement possible à la SAE d'offrir un choix de placement au jeune. Cependant, il existe d'autres façons de l'impliquer dans le processus et de lui permettre de prendre certaines décisions. Par exemple, l'informer des étapes que l'agence suivra pour le placer, l'inclure et l'inviter à assister à la planification de son placement. Une étude de Taber et Proch (1987) révèle que l'implication du jeune dans la planification de 
son placement est une méthode qui a augmenté la réussite des placements et, conséquemment, leur stabilité.

\section{L'étiquetage}

Les jeunes affirment que l'étiquette d' "enfant placé " a une connotation péjorative.

Premièrement, les jeunes n'aiment pas qu'on parle d'eux en disant «l'enfant placé » ou the foster child. C'est une étiquette dévalorisante pour ces jeunes. Selon eux, ce terme désigne une chose plutôt qu'une personne. De plus, l'utilisation de cette expression accentue leur sentiment d'être différents des autres jeunes.

"La t.s. disait toujours the foster child. Je me sentais mal, comme pas être une personne." - "Je ne me sentais pas accepté quand ils disent : ça, c'est mes enfants, et ça, c'est mon foster child. Ça faisait dix ans que je demeurais avec eux. Il me semble qu'ils auraient pu me considérer comme leur enfant. »

Par ailleurs, lorsqu'on aborde le sujet de l'acceptation du jeune dans le foyer d'accueil, les jeunes interrogés mentionnent un sentiment de rejet; ils ressentent de l'injustice face à la façon dont ils sont traités, comparativement aux enfants biologiques de la famille d'accueil. Ce constat est confirmé lors d'un forum pour les jeunes admis en placement. Des jeunes âgés de 15 à 20 ans ont aussi exprimé un mécontentement par rapport à l'étiquette d'enfant placé et un sentiment de rejet par rapport aux autres enfants dans la famille (Rice et McFadden 1988). De surcroît, le favoritisme qui semble se manifester envers les enfants biologiques rappelle qu'ils ne sont pas chez eux.

"Ils nous font sentir à part de leurs enfants. Je ne me suis jamais sentie acceptée. 》— "Si la famille a son propre enfant, elle traite lui plus spécial que l'autre personne. Il peut $y$ avoir du favoritisme puis ils sont moins portés à nous écouter quand son fils ou sa fille a un problème." "Quand leur enfant a mal à un 
bras, il va rester à la maison. Puis moi, j'avais une grosse infection de gorge et elle n'a pas voulu que je reste à la maison. Il a fallu que je marche jusqu'au centre médical pour me faire soigner. Elle avait l'auto à part de ça."

En somme, l'étiquette " enfant placé » nuit au bien-être des jeunes, leur rappelant qu'ils ne sont pas des membres à part entière de la famille d'accueil. Ils se sentent différents et parfois rejetés. Ils veulent faire partie de la famille et se sentir chez eux. Les adolescents ont besoin de sentir qu'ils appartiennent à une famille. Cette perception d'appartenance va influencer plusieurs aspects de leur vie tels que leur estime de soi et leurs comportements (Chubb et Fertman 1992 : 387).

\section{Conclusion et pistes d'intervention}

L'analyse qui précède suggère quelques pistes d'intervention; les entretiens offrent ainsi des indications qui peuvent servir aux personnes et aux organismes qui interagissent avec les jeunes.

\section{Sélection et formation en fonction des caractéristiques individuelles désirées par les jeunes}

Selon les jeunes, les caractéristiques individuelles des travailleurs sociaux, des parents d'accueil ainsi que du personnel du foyer de groupe jouent un rôle important dans le bien-être des jeunes placés à long terme.

Une attitude positive de la part d'un intervenant transmet de la confiance et des pensées positives.

La disponibilité contribue au développement d'un sentiment de sécurité; les jeunes placés en famille substitut ont besoin de savoir qu'ils peuvent compter sur quelqu'un.

Les jeunes mentionnent l'importance pour les travailleurs sociaux et les parents d'accueil d'avoir des caractéristiques, attitudes 
et comportements semblables à ceux d'un "vrai parent ". Bref, les jeunes interrogés indiquent que les intervenants sont leurs parents et ils s'attendent qu'ils agissent en tant que tels.

En dernier lieu, les jeunes signalent qu'ils ont besoin d'une personne qui va se préoccuper d'eux, et ce, de façon sincère. Les jeunes de la SAE ne veulent pas que leur "parent " (la SAE) les considère comme une simple tâche à accomplir. Ils ont besoin de savoir qu'il y a une sollicitude authentique et un souci pour leur bien-être.

\section{Assurer une certaine stabilité}

L'importance de la stabilité est manifeste dans les recherches et aussi dans les commentaires des jeunes. Celle-ci s'applique à plusieurs domaines de la vie : famille substitut, liens de parenté, environnement et milieu scolaire. Une certaine continuité dans les relations avec la famille naturelle du jeune peut favoriser la stabilité, tout comme le fait, aussi mentionné par les participants, d'être placés dans un foyer dans leur quartier d'origine ou de garder des contacts avec ce quartier. Avoir le même travailleur social pendant plusieurs années favorise aussi, selon les jeunes, leur stabilité et leur bien-être. Malheureusement, les situations particulières de chacun et la limite des ressources de placement de la SAE ne permettent pas toujours ce type de stabilité. Par contre, lorsqu'il n'est pas possible de maintenir un placement ou de faire en sorte que le même travailleur social sera toujours impliqué avec le jeune, il est nécessaire de trouver des moyens qui vont permettre une certaine continuité et un aspect de familiarité pour l'adolescent.

\section{Bien préparer les jeunes pour les changements}

Les jeunes placés à long terme sont à risque de vivre de nombreux déplacements. Compte tenu de cette réalité, il importe de bien les préparer aux changements importants dans leur vie. Lors d'un déplacement, la préparation apporte de la sécurité et permet au jeune une période d'adaptation, en plus d'en diminuer les risques (Steinhauer 1991). 


\section{Impliquer les jeunes dans le processus}

Les jeunes désirent se faire traiter comme des individus qui ont des capacités (Bélanger 1981 : 23). Les jeunes placés en foyer d'accueil aiment être impliqués dans les décisions à leur sujet (Johnson,Yoken etVoss 1995 : 972). De plus, leur implication dans les décisions de placement augmente la stabilité de leur placement (Taber et Proch 1987). Ils nous indiquent que ce sont des décisions qui les affectent, que c'est donc leur droit d'y participer ou, du moins, de faire entendre leurs opinions. Puisque ces jeunes sont souvent retirés involontairement de leur famille naturelle et que les organismes prennent des décisions sans nécessairement les consulter, ils se sentent fréquemment impuissants face aux

"...l'implication du jeune signifie la possibilité pour lui de s'impliquer dans les événements qui le concernent, d'avoir la possibilité de partager ses idées et ses opinions et de faire certains choix." situations qui les affectent. Donc, l'implication du jeune signifie la possibilité pour lui de s'impliquer dans les événements qui le concernent, d'avoir la possibilité de partager ses idées et ses opinions et de faire certains choix. Bref, cette implication lui permettrait de réaliser une démarche d'empowerment, de maturation et de responsabilisation.

En somme, impliquer les jeunes de la SAE dans le processus décisionnel est un outil de valeur non seulement face au placement, mais aussi sur le plan scolaire et sur celui des relations avec les adultes. Ainsi, en plus des apprentissages qu'ils effectueront en participant à ce processus de planification et de décision, ils auront l'occasion de s'exprimer et de se sentir un peu plus en contrôle de leur propre situation.

Ayant donné la parole aux jeunes, cette recherche qualitative et exploratoire apporte une autre perspective et ajoute des renseignements pertinents à notre connaissance des défis liés à la protection de l'enfance. La lecture des commentaires des jeunes fait surgir des pistes intéressantes d'intervention qui peuvent favoriser leur développement et améliorer nos services.

\section{Bibliographies}

BACHELOR,A. \& HORVATH,A. (1999). «The therapeutic relationship», in Hubble, M.A., Duncan, B. L. \& Miller, S. D. (eds) (1999). The Heart and Soul of Change:What works in therapy, Washington: American Psychological Association. 
BAKER, M. (1995). Child Protection, Family Violence, and Substitute Care, Canadian Family Policies: Cross National Comparisons. 236-290.

BÉLANGER, Robert (1987). Parents en perte d'autorité, Québec, Les Éditions Robert Bélanger.

BÉLANGER, Robert (1981). Parents d'adolescents, Ottawa, Les Éditions Robert Bélanger.

BERRY, Marianne et Leroy H. PELTON (1994). "Has Permanancy Planning Been Successful?» Controversial Issues in Child Welfare, E. GAMBRIL and T.J. STEIN, (eds) U.S. Allyn and Bacon, 261-274.

BOURGAULT, Denise et Françoise de la HARPE (1988). L'enfant en famille d'accueil, St-Hyacinthe, edisem.

BROAD, B. (1999). «Young people leaving care: Moving Towards. Joined Up Solutions?» Children E Society, Volume13 (2), 81-93.

BULlETIN ERAE (1997). Projet: Évaluation des résultats de l'aide à l'enfance Plantagenet, doc. mineo.

BUSH, Malcolm, Andrew C. GORDON et Robert LEBAILLY (1977). «Evaluating Child Welfare Services: A Contribution from the Clients», Social Service Review, 51, 491-501.

CANADA, Groupe de travail fédéral et provincial sur l'information sur les services à l'enfance et à la famille (1994). Bien-être de l'enfance au Canada, Ottawa, Ministère des Approvisionnements et Services Canada.

CHUBB, Nancy H. et FERMAN, Carl I.(1992). «Adolescents' Perceptions of Belonging in Their Families», Families in Society: The Journal of Contemporary Human Services, Sept., 387-394.

COOK, R.J. (1994). "Are we helping foster care youth prepare for their future?" Children and youth services, 16 (314), 213-229.

DUNCAN, B. L., \& Scott D. MILLER (2000). The Heroic Client: Doing client-directed, outcome-informed therapy, San Francisco: Jossey-Bass

GOLDSTEIN, Joseph, Anna FREUD and Albert J. SOLNIT (1978). Dans l'intérêt de l'enfant?, Paris, ESF.

HANIGAN, Patricia (1990). La jeunesse en difficulté : comprendre pour mieux intervener, Québec, Presses de l'Université du Québec.

HUBBLE, M. A., DUNCAN, B. L. \& MILLER, S. D. (eds) (1999). The Heart and Soul of Change: What works in therapy, Washington: American Psychological Association.

JOHNSON, Penny R.,YOKEN, C. etVOSS, R. (1995). “Family Foster Care Placement:The Child's Perspective", Child Welfare, 74: 5,959-974.

KIRSCH, I. (1999). How Expectancies Shape Experience, Washington: American Psychological Association.

KORBIN, Jill (1977). "Anthropological Contributions to the Study of Child Abuse”, Child Abuse and Neglect, 1, 7-24.

KUFELDT, K., ARMSTRONG, J. et DOROSH, M. (1995). "How Children in Care View Their Own and Foster Families", Child Welfare,74, 695-715.

LE BOSSÉ,Yvan (1996). «Empowerment et pratiques sociales : illustration du potentiel utopie prise au sérieux ", Nouvelles pratiques sociales, 9:1,127- 145.

LOI SUR LES SERVICES À L'ENFANCE ET À LA FAMILLE, L.R.O. 1984, chap. 55.

MAGURA, Stephen (1982). «ClientsView Outcomes of Child Protective Services», Social Casework, 63: 9, 522-531. 
MAYER, Robert et OUELLET, F. (1991). Méthodologie de recherche pour les intervenants sociaux, Boucherville, Gaëtan Morin.

MCKENZIE, Brad (1994). "Current Issues in Foster Family Care». Current Perspectives on Foster Family Care for Children and Youth, Ontario, Wall \& Emerson, Inc., 1-17.

MILLER, S. D., Duncan, B. L., \& Hubble, M. A. (1997). Escape from Babel:Toward a unifying language for psychotherapy practice, New York: Norton.

MILNER, Joel S. et PELTON, Leroy H. (1994). "Is poverty a Key Contributor to Child Maltreatment?" Controversial Issues in Child Welfare, Eileen GAMBRILL et Theodore J. STEIN, (eds) United-States, Allyn and Bacon, 16-28.

NATIONAL RESEARCH COUNCIL,(1993). Understanding Child Abuse and Neglect, Washington D.C., National Academy Press.

ONTARIO, Ministry of Community and Social Services (1985a). A Policy Statement on Standards for Foster Care, Ontario, MCSS.

ONTARIO, Ministère des services sociaux et communautaires (1985b). Principaux aspects de la Loi sur les services à l'enfance et à la famille, Ontario, MSSC.

PATTON, (1990). Qualitative Evaluation and Research Methods, United States, Sage Publications, Inc.

RICE, Dale L. et McFADDEN, Emily Jean (1988). "A Forum for Foster Children», Child Welfare, 67: 3, 231-243.

SELIGMAN, Martin E.P.,(1993). "What You Can Change...And What You Can't: The Complete Guide to Successful Self-Improvement". New York, Fawcett Columbine.

SHULMAN,Lawrence (1993). "Developing and Testing a Practice Theory:An Interactional Perspective", Social Work, 38, 91-97.

SNYDER, C. R., Michael, S.T., \& Cheavens, J. (1999). "Hope as a psychotherapeutic foundation for nonspecific factors, placebos, and expectancies”, in M.A. Huble, B. Duncan, \& S. Miller (eds.), Heart and soul of change, Washington, DC. American Psychological Association.

STARR, Raymond H. Jr. (1988). "Physical Abuse of Children", Handbook of Family Violence, New York, Plenum.

STEINHAUER, Paul (1991). The Least Detrimental Alternative: A Systematic Guide to Case Planning and Decision Making for Children in Care, Toronto, University of Toronto Press.

TABER, Merlin et PROCH, Kathleen (1987). "Placement Stability for Adolescents in Foster Care: Findings from a Program Experiment". Child Welfare, 66: 5, 433-445.

TROCMÉ, Nico, McPHEE, Debra et TAM, Kwok Kwan (1995). "Child Abuse and Neglect in Ontario Incidence and Characteristics”, Child Welfare,74(3),563-586.

\section{Notes}

1. Cette recherche a été effectuée dans le cadre d'un mémoire de maitrise en service social à l'Université d'Ottawa sous la direction de Marie Drolet.

2. Dans le présent document, le genre non marqué s'applique à la fois aux hommes et aux femmes. 